\title{
MICROMACHINED HIGH-ASPECT-RATIO PARYLENE BEAM AND ITS APPLICATION TO LOW-FREQUENCY SEISMOMETER
}

\author{
Yuji Suzuki* and Yu-Chong Tai** \\ *Dept. Mechanical Engineering., The University of Tokyo, Tokyo 113-8656, JAPAN \\ **Electrical Engineering, California Institute of Technology, 136-93, Pasadena, CA 91125, U.S.A. \\ E-mail: ysuzuki@thtlab.t.u-tokyo.ac.jp
}

\begin{abstract}
A new microfabrication technology for high-aspectratio Parylene structure is developed for soft spring applications and applied to in-plane seismometer which covers low frequency $(<1 \mathrm{~Hz})$ and small acceleration range. Parylene beams having $10-40 \mu \mathrm{m}$ wide and an aspect ratio of 10-30 were successfully fabricated. Since Parylene has a small Young's modulus and is non-brittle material, high-aspect-ratio robust beam having spring constant on the order of $1 \times 10^{-3} \mathrm{~N} / \mathrm{m}$ was realized. A prototype capacitive seismometer was also made, and its resonant frequency and noise spectral density was respectively measured to be $37 \mathrm{~Hz}$ and $45 \mu \mathrm{g} / \sqrt{\mathrm{Hz}}$. Since the Brownian noise is only $25 \mathrm{ng} / \sqrt{\mathrm{Hz}}$, seismometer having much lower noise floor may be feasible using this new technology.
\end{abstract}

\section{INTRODUCTION}

For mechanical transducers, sensitivity to mechanical force should vary by orders of magnitude depending on the direction. The structure should be soft in the direction of interest, but it should be very rigid to other two perpendicular directions to minimize mechanical cross talk. Therefore, fabrication technologies for highaspect ratio structures (HARS) attract much attention in the MEMS field.

The most straightforward approach for HARS is to use deep reactive ion etching (DRIE) of silicon substrate such as the Bosch process [1]. HEXSIL[2, 3] and HARPSS[4] are also proposed for high-aspect polysilicon structures, where deep trenches are refilled with polysilicon. However, both single crystal silicon and polysilicon are brittle so mechanical failure is of concern. High-aspect ratio metal structures can be fabricated by LIGA[5] process, but it requires X-ray, which is generally a much more complex process. Moreover, the existing technologies all use material having a large Young's modulus $>100 \mathrm{GPa}$, submicron lithography is often required to realize soft springs [6].
The objective of the present study is then to develop a new microfabrication technology to produce high-aspect ratio Parylene beams with small spring constant. The advantage of using Parylene is twofold. First, Parylene has a small Young's modulus ( 4GPa), which makes easy small spring design. Secondly, Parylene is a non-brittle material with a large linear-elastic range (yield strain $\sim 3 \%$ ), which allows large deflection without failure. Here, we present the first in-plane seismometer using the Parylene high-aspect ratio beams.

\section{FABRICATION}

The major process flow for the high-aspect-ratio Parylene beam fabrication is shown in Fig. 1. The process starts with 4 " silicon wafers having $2 \mu \mathrm{m}$ thermal oxide. The oxide is patterned on the front side, and the substrate is DRIE etched with $400 \mu \mathrm{m}$-deep trenches. The trenches are used as Parylene mold and their depth defines the desired height of the beams. Then, a second $2 \mu \mathrm{m}$ thermal oxide is grown on all exposed silicon surface as the etch-stop layer for the later DRIE. This is followed by a Parylene-C deposition with a thickness of $10-20 \mu \mathrm{m}$ to refill the trenches. The deposition pressure is $22 \mathrm{mT}$. The Parylene film is then etched back using $\mathrm{O}_{2}$ plasma. Next, the backside oxide is patterned and silicon is etched away from the backside with DRIE to free the Parylene beams, although covered by the thermal oxide. Finally, the beams are completely freed by BHF etch of the etch-stop oxide.

Figure 2a shows an SEM image of the $30 \mu \mathrm{m}$-width trench after the etch back of Parylene film. Clearly, the Parylene deposition is conformal, and film thickness inside the trench is almost uniform. Also seen is small void inside, which is caused because the top part of the trench is sealed at the early stage of the deposition. If desirable, this void can be completely eliminated with an etchback and a second Parylene deposition (Fig. 2b).

\section{PARYLENE HIGH-ASPECT RATIO SPRING}

Figure 3a shows an SEM image of a freestanding 
a)

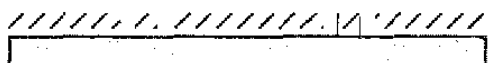

b)

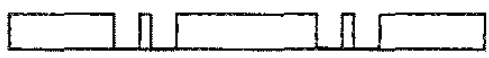

c)

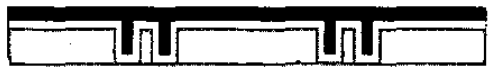

d)

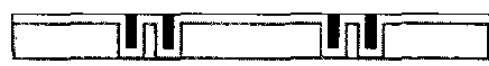

e)

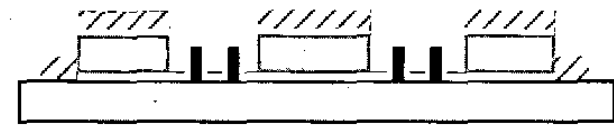

f)

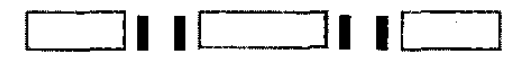

Figure 1: Process flow for the high-aspect-ratio Parylene structure. a) Wet oxidation and pattern oxide, b) Make trench by DRIE and 2nd wet oxidation, c) Deposit 10$20 \mu$ m-thick Parylene-C, d) Etch back Parylene with $\mathrm{O}_{2}$ plasma, e) Pattern oxide backside and etch through using DRIE, f) Etch oxide and release Parylene structures.
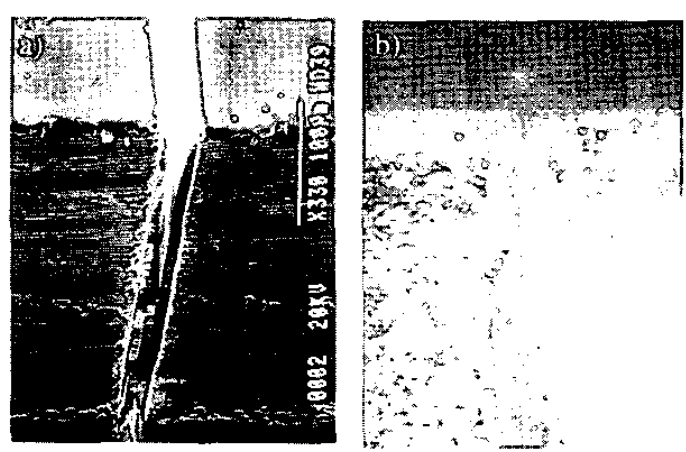

Figure 2: $30 \mu \mathrm{m}$-width trench. a) SEM image after the etch back, b) Photograph of cross section after the second deposition.

beam having $30 \mu \mathrm{m}$ in width, $400 \mu \mathrm{m}$ in height, and $2.5 \mathrm{~mm}$ in length. Triangle-shaped trench etched into the substrate works well for the anchor. Figure 3 b shows a magnified view of a pendulum structure with a $10 \mu \mathrm{m}$ wide beam (aspect ratio 30). The device was fixed onto a gonio stage and the deflection of the pendulum due to the gravity force was measured by changing the tilt angle. Figure 4 shows the deflection of the beam versus the gravity force. The spring constant is as low as

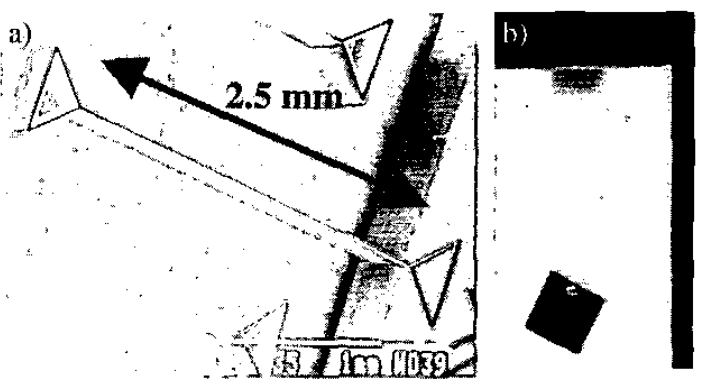

Figure 3: Pendulum using Parylene beam. a) SEM images of high-aspect ratio beam and its anchor, b) pendulum structure using $10 \mu \mathrm{m}$ width beam suspending $800 \times 800 \mu \mathrm{m}$ Si substrate.

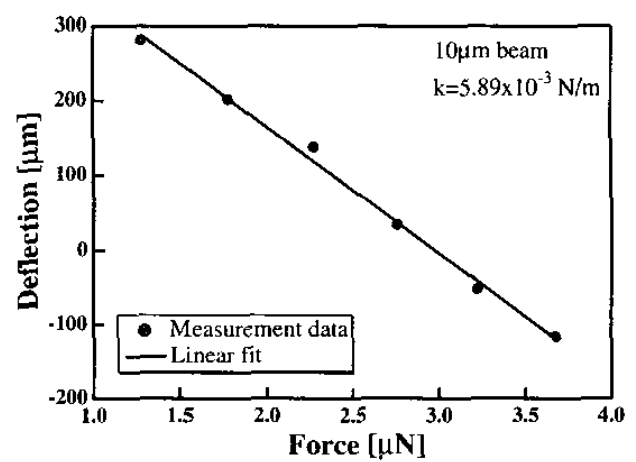

Figure 4: Spring constant measurement of $10 \mu \mathrm{m}$-width beam.

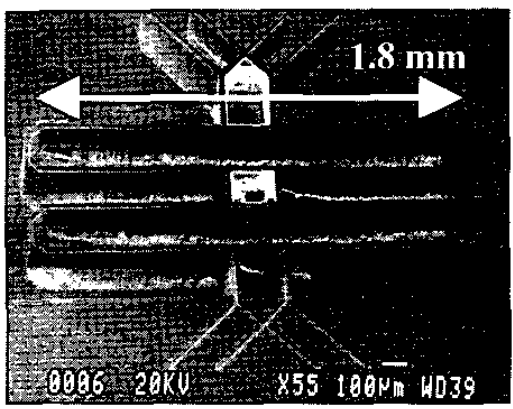

Figure 5: SEM image of leaf spring structure using Parylene beam of $20 \mu \mathrm{m}$ in width and $400 \mu \mathrm{m}$ in height.

$0.0059 \mathrm{~N} / \mathrm{m}$, a value smaller than the designed value. This is partially because the second deposition of Parylene was not made for this test device, and the cross section of the beam is U-shaped as shown in Fig. 2a. It is noted that this soft spring is also robust and will not fracture even by shaking vigorously.

In order to study the dynamic response of the Parylene beams, a $1.75 \times 1.75 \mathrm{~mm}$ silicon seismic mass supported with two Parylene leaf springs (Fig. 5\&6) was fabricated. The beams are $20 \mu \mathrm{m}$ in width and $400 \mu \mathrm{m}$ in height. The device was glued onto a loud 
a)

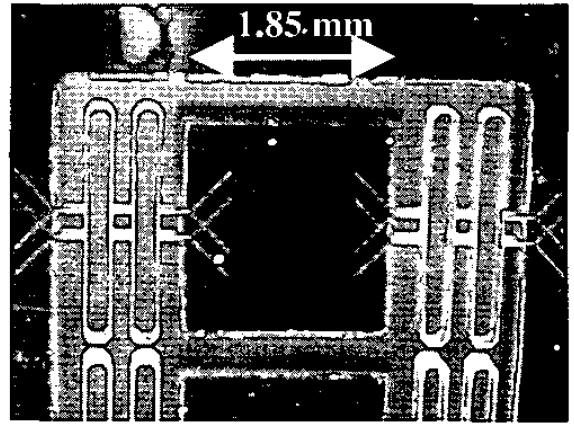

b)

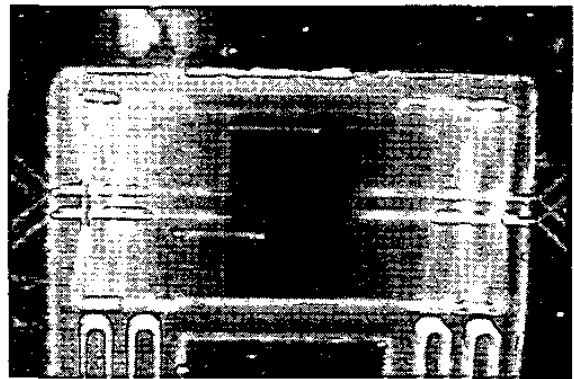

Figure 6: a) Oscillator prototype having two leaf springs and $1.75 \times 1.75 \mathrm{~mm}$ mass in between. b) Oscillation at the resonant frequency $(\sim 113 \mathrm{~Hz})$

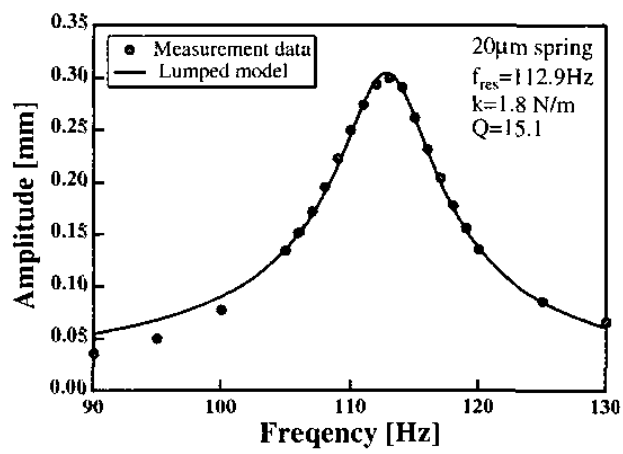

Figure 7: Frequency response of the oscillator.

speaker and shaken in the in-plane direction at prescribed frequency and amplitude. The relative displacement of the seismic mass to the substrate was measured visually using a CCD camera. The CCD camera was equipped with a high magnification lens, and one pixel of the image corresponds to about $3 \mu \mathrm{m}$. As shown in Fig. 7, the measurement data fit well with a simple spring-mass-damper model. and the resonant frequency $f_{\text {res }}$ and quality factor $Q$ are determined to be $112.9 \mathrm{~Hz}$ and 15,1 , respectively. The peak-to-peak amplitude at the resonance is as large as $600 \mu \mathrm{m}$, and the neighboring leaf springs are almost attached to each other as shown in Fig. 7. It is noted that the characteristic of these leaf springs remains unchanged even after being driven at their resonant frequency oscillation for sev- a)
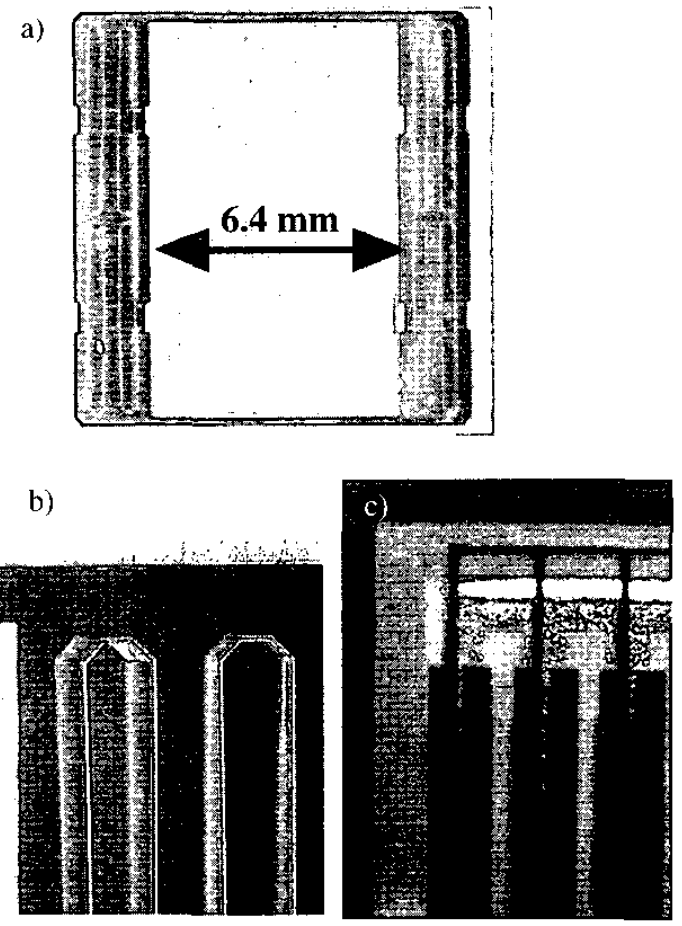

d)

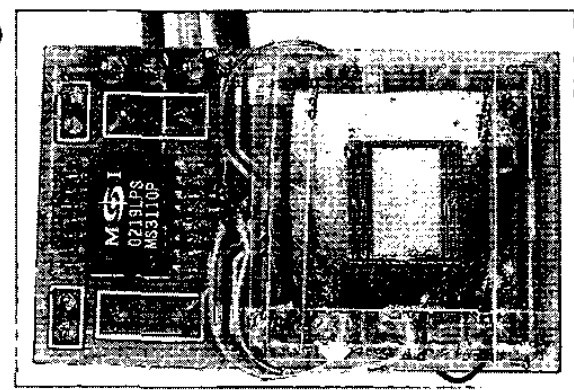

Figure 8: Prototype of in-line capacitive seismometer using high-aspect-ratio Parylene beam. a) Seismic part, b) Magnified view of the leaf spring $(20 \mu \mathrm{m}$ wide, $400 \mu \mathrm{m}$ high), c) Magnified view through the counter electrode, d) Prototype device with a readout IC.

eral hours, which corresponds to about $2 \times 10^{6}$ cycles.

\section{PROTOTYPE IN-PLANE SEISMOMETER}

In order to demonstrate the advantage of the Parylene high-aspect ratio beam, prototype of in-plane capacitive seismometer was designed and fabricated. As shown in Figs. 8a\&b, the seismic mass $(9.4 \times 6 \mathrm{~mm})$ is supported with four leaf springs and the electrical contact was made through a $\mathrm{Cr} / \mathrm{Au} / \mathrm{Cr}$ layer $(100 \AA / 2000 \AA /$ $100 \AA$ ) thermally-deposited on the top of the $20 \mu \mathrm{m}$ width beams. Another Parylene film of $2 \mu \mathrm{m}$ in thick- 


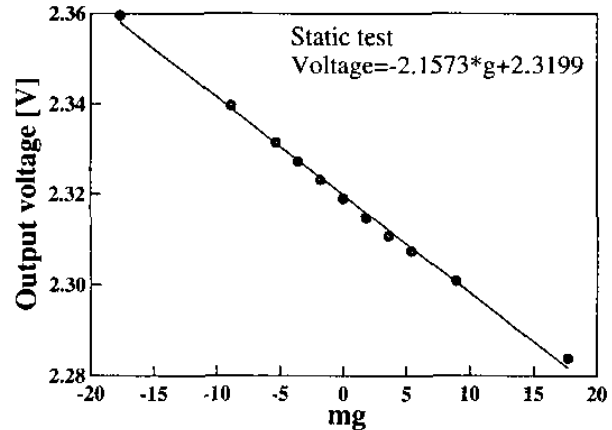

Figure 9: Static response of the prototype seismometer.

ness was deposited on the top of the electrode as the protection layer. The resistance between the substrate and the grid electrodes is $60 \Omega$. The resonant frequency measured is $37 \mathrm{~Hz}$, which is in good agreement with the designed value of $35 \mathrm{~Hz}$. The displacement of the seismic mass was measured by the capacitance change. Counter electrodes were patterned on a glass wafer and attached to the silicon substrate with a gap of about $100 \mu \mathrm{m}$ as shown in Fig. 8c. Figure $8 \mathrm{~d}$ shows our early prototype seismometer, in which a differential-type readout IC (MicroSensors Inc., MS3110) having a low noise floor of $4 \mathrm{aF} / \sqrt{\mathrm{Hz}}$ is used. Figure 9 shows the static response of the seismometer using a tilt experiment. The sensitivity of this prototype is only $0.221 \mathrm{~V} /$ $\mathrm{g}$, which is 5 times smaller than the designed value. This is probably due to the parasitic capacitance, since the gap between electrodes is not small enough when compared with the electrode width. Since the noise floor of the voltage output is about $10 \mu \mathrm{V} / \sqrt{\mathrm{Hz}}$, the noise spectral density is $45 \mu \mathrm{g} / \sqrt{\mathrm{Hz}}$. Note that, our second generation prototype under development has lower resonant frequency, improved electrode design, and better readout IC settings, and the noise floor of about $1 \mu \mathrm{g} / \sqrt{\mathrm{Hz}}$ can be expected with a bandwidth of $20 \mathrm{~Hz}$.

The Brownian equivalent acceleration noise $g_{n, B}$ is given by

$$
g_{n, B}=\frac{1}{g} \sqrt{\frac{8 \pi k T f_{\text {res }}}{M Q}}[\mathrm{~g} / \sqrt{\mathrm{Hz}}],
$$

where $k . T$, and $M$ are respectively the Boltzman constant, temperature, and the weight of the seismic mass [7]. In the present seismometer, $f_{\text {res }}=37 \mathrm{~Hz}$, which is the lowest resonant frequency among MEMS seismometers ever built. Moreover, the mass $M=66 \mathrm{mg}$ is much larger than the mass of previous seismometers.
Thus, $g_{n, B}$ given by Eq. (1) is as low as $25 \mathrm{ng} / \sqrt{\mathrm{Hz}}$, and three orders of magnitude smaller than the noise floor measured. Therefore, the noise level of the present seismometer is not be limited by the Brownian noise, and there exists much room to improve its sensitivity.

\section{CONCLUSIONS}

A new high-aspect-ratio Parylene beam technology is developed. Soft but robust spring is successfully fabricated and fully characterized by a series of experiments. Early prototype of capacitive in-line seismometer having a record-low resonant frequency $(37 \mathrm{~Hz})$ was also made. The noise floor is found to be $45 \mu \mathrm{g} / \sqrt{\mathrm{Hz}}$, and is not limited by the Brownian noise.

\section{ACKNOWLEDGMENTS}

This work is supported by the NSF Engineering Research Center (ERC) at Caltech. YS acknowledges Professor N. Kasagi in the University of Tokyo for his support during the course of this work.

\section{REFERENCES}

[1] Ayon, A. A., Braff, R., Lin, C. C., Sawin, H. H., and Schmidt, M. A., "Characterization of a time multiplexed inductively coupled plasma etcher," J. Electrochem. Soc., 146, (1999), pp. 339-349.

[2] Keller, C., and Ferrari, M., "Milli-scale polysilicon structures," Proc. Solid-state Sensor and Actuator Workshop, Hiton Head, (1994), pp. 132-137.

[3] Horsley D.A., Cohn, M. B., Singh, A., Horowitz, R., and Pisano, A. P., "Design and fabrication of an angular microactuator for magnetic disk drives," $J$. Microelectromech. Syst., 7, (1998), pp. 141-148.

[4] Ayazi, F., and Najafi, K., "High aspect-ratio combined poly and single-crystal silicon (HARPSS) MEMS technology," J. Microelectromech. Syst., 9, (2000), pp. 288-294.

[5] Guckel, H., "High-aspect-ratio micromachining via deep X-ray lithography," Proc. IEEE, 86, (1998), pp. 1586-1593.

[6] Weigold, J. W., Najafi, K., and Pang, S. W., "Design and fabrication of submicrometer, single crystal Si accelerometer," J. Microelectromech. Syst., 10, (2001), pp. 518-524.

[7] Bernstein, J., Miller, R., Kelley, W., and Ward, P., "Low-noise MEMS vibration sensor for geophysical applications," Proc. Solid-State Sensor and Actuator Workshop, Hilton Head, (1998), pp. 55-58. 